\title{
Erosion/re-deposition modeling in an ITER divertor-like high-density, low-temperature plasma beam
}

\author{
G.A. van Swaaij ${ }^{1}$, A. Kirschner ${ }^{2}$, D. Borodin ${ }^{2}$, W.J. Goedheer ${ }^{1}$, \\ K. Bystrov ${ }^{1}$, G. De Temmerman ${ }^{1}$ \\ ${ }^{1}$ FOM Institute DIFFER - Dutch Institute for Fundamental Energy Research, \\ Association EURATOM-FOM, Partner in the Trilateral Euregio Cluster, PO. Box \\ 1207, 3430 BE Nieuwegein, the Netherlands \\ ${ }^{2}$ Institute for Energy and Climate Research - Plasma Physics, Forschungszentrum \\ Jülich GmbH, Association EURATOM-FZJ, Partner in the Trilateral Euregio \\ Cluster, 52425, Jülich, Germany
}

\begin{abstract}
Transport of hydrocarbon impurities in a high-density $\left(>10^{20} \mathrm{~m}^{-3}\right)$, low-temperature $(<2 \mathrm{eV})$ plasma beam was studied with the ERO code. The high ion density and low temperature cause strong Coulomb collisionality between plasma ions and impurity ions. The collisionality is so strong that ions typically do not complete their Larmor orbits. The high collisionality causes impurity entrainment: impurity ions quickly acquire a velocity close to the plasma flow velocity. This causes a relatively high surface impact energy: the calculated mean impact energy of $\mathrm{CH}_{x}$ was $8.1 \mathrm{eV}$ in a plasma with $\mathrm{T}_{e}=0.7 \mathrm{eV}$. Simulation results were compared to an $a-\mathrm{C}: \mathrm{H}$ erosion experiment in the linear plasma generator Pilot-PSI. The large uncertainties in literature values for the sticking probability of hydrocarbon radicals are shown to cause a serious uncertainty in the calculated re-deposition pattern. In contrast, the radial electric field component perpendicular to the axial magnetic field lines did not have a major effect on the redeposition profile.
\end{abstract}




\section{Introduction}

In ITER and in future tokamak reactors, effective erosion of the divertor wall caused by the intense particle heat fluxes will be a major issue. Physical sputtering is caused if the ion energy exceeds the sputtering threshold, and chemical sputtering is caused for wall materials that react with hydrogenic ions, such as carbon and beryllium. The impurities thus originating from the wall will be transported through the plasma and subsequently re-deposited at another site. In ASDEX-Upgrade, it was found that the outer divertor is a net erosion area, and that a large fraction of the eroded carbon is transported to the inner divertor, which is a net deposition area [1]. Re-deposition occurs also in shadowed areas; in both cases, it can lead to layer growth and subsequent hydrogen retention, possibly limiting the duration of D-T campaigns [2]. On the other hand, re-deposition at or very nearby the site of erosion can actually suppress the erosion, reducing the net erosion. Impurity transport to the plasma core causes dilution of the plasma and radiative cooling, hampering fusion performance and even potentially causing disruptions. Understanding impurity transport is therefore of great importance.

The plasma close to the ITER divertor strike point is predicted to have low electron and ion temperatures $\left(T_{e} \approx T_{i}<5 \mathrm{eV}\right.$, necessary to limit the heat flux to the plasma facing components), and a very high density $\left(n_{e}>10^{20} \mathrm{~m}^{-3}\right)$ [3]. In this plasma parameter regime, ion-ion Coulomb collisions are extremely efficient. For example, in an ITER-divertor-like plasma with $T_{e}=2 \mathrm{eV}$ and $n_{e}=5 \cdot 10^{20} \mathrm{~m}^{-3}$, the effective ionion collision time for Maxwell-distributed $\mathrm{D}^{+}$ions is only $0.013 \mu \mathrm{s}$ [4]. For reference, the gyration period of $\mathrm{D}^{+}$ions in a typical magnetic field of $5 \mathrm{~T}$ is slightly larger, namely $0.015 \mu \mathrm{s}$. In other words, ions do not typically complete full Larmor orbits. The high collisionality regime is, to date, only accessible in a few experiments, including the linear plasma generators Magnum-PSI [5] and its forerunner experiment Pilot-PSI [6]. A unique feature of these experiments is the extremely high electron density $\left(>10^{20} \mathrm{~m}^{-3}\right)$ of the plasma beams.

A priori, one may expect two important effects to result from the high collisionality in these experiments. Firstly, because impurities are not fixed to gyro-orbits around magnetic field lines, one may expect enhanced transport perpendicular to the B-field in a high-collisionality plasma. The importance of the electric field for the cross-field transport is not so clear. In recent simulations of tungsten migration in the C-MOD divertor [7], inclusion of a radial electric field in the calculation made no significant difference to the simulated re-deposition pattern. However, those calculations were performed for much higher $\mathrm{T}_{e}$ and lower $n_{e}$ than is expected near the ITER divertor, and it is not obvious that those findings will still hold in such a high collisionality plasma. A second effect of the high collisionality is that the impurity ion energy at the target is several times higher than one would expect by simply taking the energy that the ion acquires in the plasma sheath. This is due to entrainment of impurities with the hydrogen plasma flow [8], as was evidenced earlier in PISCES-A [9]. The present paper discusses these effects by analysis of experimental erosion/redeposition patterns and the 
results of transport simulations. Furthermore, a number of important open questions and complicating factors in this plasma regime will be identified.

Numerical impurity transport modeling with the ERO code $[10,11]$ is compared with an amorphous carbon $(a-\mathrm{C}: \mathrm{H})$ erosion experiment in Pilot-PSI. The erosion profile after exposure was measured by interferometry, as discussed in section 2. General results of the transport simulation are shown in section 3. Since redeposition of the eroded carbon is strong, the simulated erosion profile depends strongly on the surface sticking probability. A brief overview of sticking probabilities found in the literature is presented in section 4 , and in section 5 these sticking probabilities are used to compare experimental and simulated erosion profiles. There, the results obtained with various parameter sets are compared, in order to give an overview of the most important errors inherent to this type of analysis.

\section{Experiment}

Prior to the erosion experiment, a homogeneous $a-\mathrm{C}: \mathrm{H}$ layer was deposited on top of a polished aluminum target. Deposition was performed in Pilot-PSI by injecting methane $\left(\mathrm{CH}_{4}\right)$ during 7.5 minutes into unmagnetised expanding thermal argon plasma, through a perforated ring placed closely behind the cascaded arc plasma source. The methane is dissociated by the argon plasma and the thusly created radicals are deposited on the target, which is placed downstream. This setup closely resembles that of [12], though the source design and experiment geometry are slightly different, and the reactant gas here was methane rather than acetylene, leading to deposition of $a$-C:H, rather than a diamondlike layer. During the deposition, the cascaded arc was operated with a source current of $150 \mathrm{~A}$, with an Ar flow rate of $2.5 \mathrm{slm}$ (standard liters per minute). The external methane injection rate was $0.25 \mathrm{slm}$.

First, an $a$-C:H layer was deposited on a reference target. A mass balance was used before and after exposure, and the total deposited mass of the layer was determined to be $1.04 \pm 0.12 \mathrm{mg}$. After measuring the reference sample, a fresh target was inserted into Pilot-PSI, on which an identical $a-\mathrm{C}: \mathrm{H}$ layer was deposited. This second target was then used for the erosion experiment. It was exposed during 10 seconds to a magnetised hydrogen plasma jet with $B=0.4 \mathrm{~T}$. During this exposure, the plasma density and temperature at an axial distance of $25 \mathrm{~mm}$ from the target were measured by Thomson scattering. The results are shown in figure 1.

A photograph of the target after exposure to the hydrogen plasma jet is shown in figure 2. Despite the exposure time of only 10 seconds, the layer had already been eroded away completely at the center of the beam. The mass remaining of this target after deposition and erosion was $0.80 \pm 0.15 \mathrm{mg}$. Subtracting this mass from the mass of the layer on the reference target gives the estimated erosion loss: $0.24 \pm 0.19 \mathrm{mg}$.

Interferometry was used to measure the layer thickness of the target shown in

figure 2. A variable angle ellipsometer was used to perform this measurement. The ellipsometric $\Psi$ (the magnitude of the ratio between the $p$ and $s$ components of the 


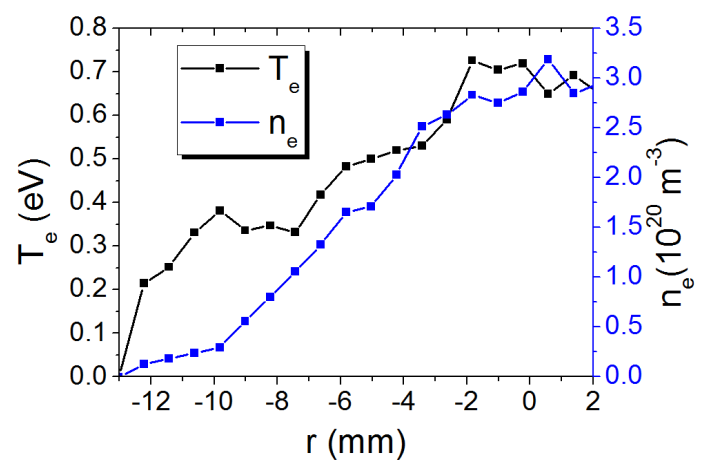

Figure 1. $n_{e}$ and $T_{e}$ profiles at $25 \mathrm{~mm}$ before the target, as measured by Thomson scattering. These are used as input for the modeling with ERO.

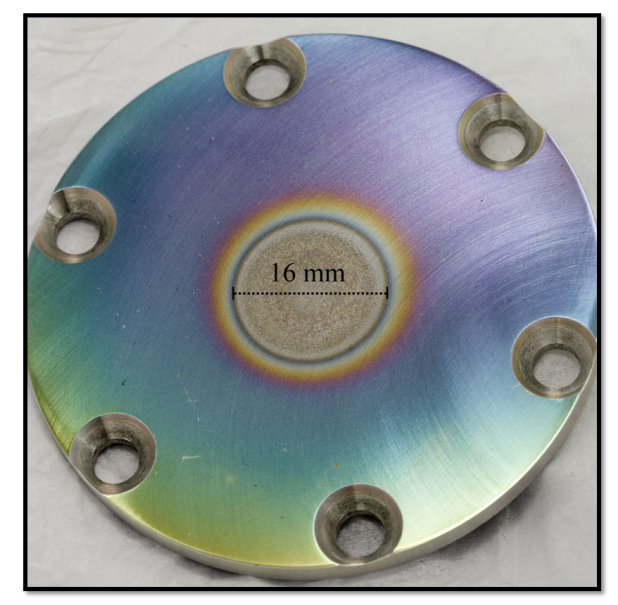

Figure 2. The target after deposition of the $a-\mathrm{C}: \mathrm{H}$ layer and subsequent erosion by $\mathrm{H}$ plasma in Pilot-PSI.

reflectivity) and $\Delta$ (its phase) were measured in a wavelength region between $245 \mathrm{~nm}$ and $1689 \mathrm{~nm}$. The angle of incidence of the polarised light was varied between $45^{\circ}$ and $85^{\circ}$. These measurements are shown in figure 3 a. The ellipsometric $\Psi$ has various interference maxima, due to constructive and destructive interference between the reflection from the top of the $a-\mathrm{C}: \mathrm{H}$ layer, and reflection from the carbon/metal interface. The wavelengths with maximum destructive interference are given by $\lambda_{\Psi \max }=\frac{4 * l}{2 k+1}$, where $l=d_{\text {layer }} * \cos \left(\theta_{\text {layer }}\right)$ is the distance travelled by the polarised light from the top of the sample to the carbon/metal interface, $\theta_{\text {layer }}$ the angle of the light inside the layer, and $k$ is an integer representing the number of the peak. This formula gives the ratios between the wavelengths of various interference peaks. Using these ratios, we determined that the peak around $500 \mathrm{~nm}$ is the destructive interference peak having $k=2$. The wavelength $\lambda_{\Psi \max }$ is shown in figure $3 \mathrm{~b}$. The angle of the light inside the a-C:H layer is given by Snell's law: $\frac{\sin \left(\theta_{\text {layer }}\right)}{\sin \left(\theta_{\text {air }}\right)}=\frac{1}{n}$, with $n$ the refractive index of the layer. Once we know $n$, these formulas enable us to calculate the layer thickness $d$.

The refractive index of $a-\mathrm{C}: \mathrm{H}$ layers depends on layer properties, such as its density. For example, in deposition experiments [13] values ranging from 1.59 to 2.13 were 

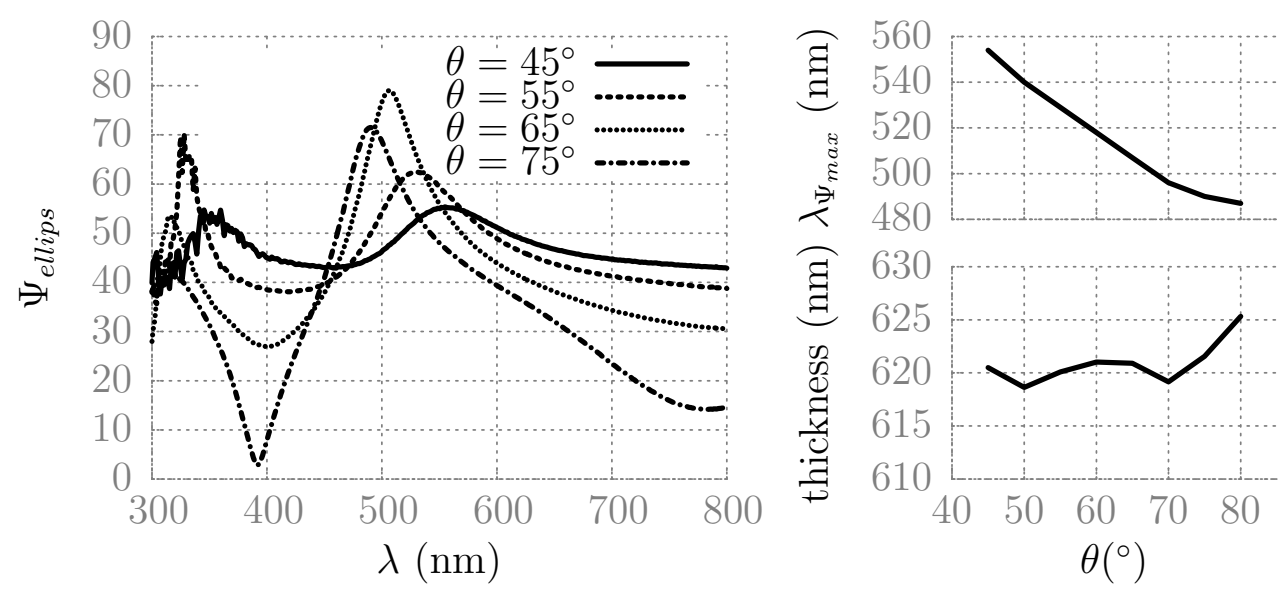

Figure 3. (left) raw measurement of ellipsometric $\Psi$, for different wavelengths $\lambda$ and angles of incidence $\theta$ of the polarised light; (right top) the resulting wavelengths of the relevant interference maximum, plotted as a function of $\theta$; (right bottom) the corresponding thicknesses that were calculated by assuming $n=1.57$.

measured in layers with densities ranging from $0.80 \mathrm{~g} \mathrm{~cm}^{-3}$ to $1.51 \mathrm{~g} \mathrm{~cm}^{-3}$, respectively. In general, obtaining the most accurate ellipsometric determination of the refractive index $n$ requires an understanding of the optical properties of the $a-\mathrm{C}: \mathrm{H}$ layer. However, the fact that we know $\lambda_{\max }$ at various angles of incidence allows for a good estimation of $n$. Clearly, a correct analysis should give identical thicknesses for all incident angles. Since $n$ is not dependent on the angle of the light, the correct value of $n$ is the one for which the calculation yields an identical thickness at all the incident angles. The value for which this is the case is $n=1.57$, which is not an unusual value for a porous $a-\mathrm{C}: \mathrm{H}$ layer. The thicknesses that were calculated are shown in figure $3 \mathrm{c}$. With this value of $n$, all angles give the same value: $620 \pm 2 \mathrm{~nm}$, except for the measurement at $80^{\circ}$ incidence, where the error was largest.

These variable angle measurements were repeated at different positions along the target. The value $n=1.57$ gave good results for fitting measurements at all positions for which distance from the beam center $r$ was at least $10 \mathrm{~mm}$. For $r$ between $8 \mathrm{~mm}$ and $10 \mathrm{~mm}$, the layer was too inhomogeneous to measure with this technique. In the center of the target, with $r<9 \mathrm{~mm}$, no carbon film or other redeposits were observed at all. The thickness profiles that were thus calculated are used later in figures 8 and 9 . Dividing the measured mass difference of the reference target by the integrated deposit volume gives a layer density $\rho=0.8 \mathrm{~g} \mathrm{~cm}^{-3}$, corresponding to a porous $a$-C:H layer.

\section{Impurity transport in high-density, low-temperature plasma}

In the ERO simulations, eroded methane test particles enter the plasma with a thermal energy distribution corresponding to a source temperature of $800{ }^{\circ} \mathrm{C}$ (approximately the target temperature), with a cosine angular distribution. After entering the plasma, the molecules get ionised and dissociated predominantly through charge exchange with 
plasma ions and dissociative recombination with electrons. Reaction rates for methane breakdown come from [14]. The modeling used a static background plasma, with plasma parameters taken from the experimental Thomson scattering measurements. To verify the applicability of the modeling assumptions in Pilot-PSI conditions, experimental photon emission plumes from external methane injection have been benchmarked against simulated plumes; those results were reported elsewhere [15]. For simplicity, it was assumed that all eroded molecules enter the plasma with identical distributions of energies and angles. This allows us to greatly simplify the analysis: it enables separation of the modeling of impurity transport in the plasma and the calculation of actual redeposition profiles on the target. ERO has been used in this way previously to reproduce the deposition profile during methane injection [16], but this required careful matching of the erosion yield along the target to reproduce the experimentally observed deposition pattern.

The radial electric field component perpendicular to $\mathbf{B}$ is not easily measurable directly. In Pilot-PSI, it has been calculated from a careful analysis of measurements of the Doppler shift of hydrogen lines in the rotating plasma [17]. The resulting electric potential profile is plotted in figure 4. The maximum value of this electric field is $6.4 \cdot 10^{2} \mathrm{~V} / \mathrm{m}$, occuring $5 \mathrm{~mm}$ outside the beam center. However, a complete database of the radial electric field at all achievable Pilot-PSI conditions is, at the moment, lacking; it is not certain whether the given plasma potential applies unchanged to the present experiment. To estimate whether this uncertainty is of importance, transport simulations results both with and without radial electric field will be compared.

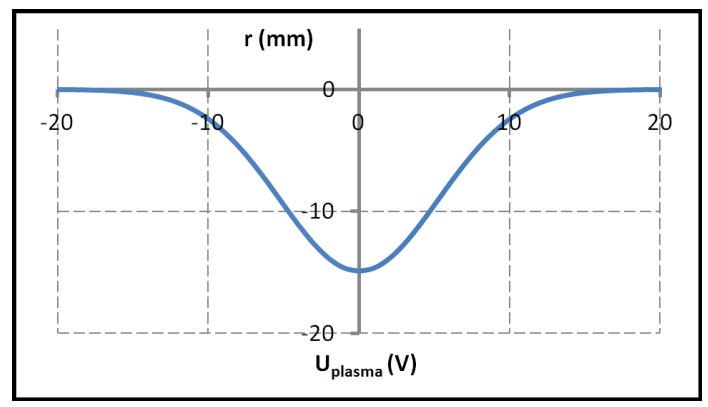

Figure 4. Electric potential profile assumed for Pilot-PSI.

The trajectories of a few $\mathrm{CH}_{x}$ test particles through the plasma are shown in figure 5. Plotted are trajectories of particles that were eroded at various locations on the target of Pilot-PSI (the target is indicated by the grey circle). In both cases, the particles leave the target as neutrals. Only the transport as ions is shown, so the plotted trajectories begin once the test particle is first ionized. The plasma column is located along the z-axis. Particles are followed until the particle either returns to the target or leaves the simulation volume, after which the simulation stops and the final location of the particle is recorded. Two cases were considered: $\mathrm{T}_{e}=0.7 \mathrm{eV}$ (equivalent to the experimental condition), and $\mathrm{T}_{e}=2 \mathrm{eV}$ (also typical for Pilot-PSI, and closer to expected values near the ITER divertor strike point). 


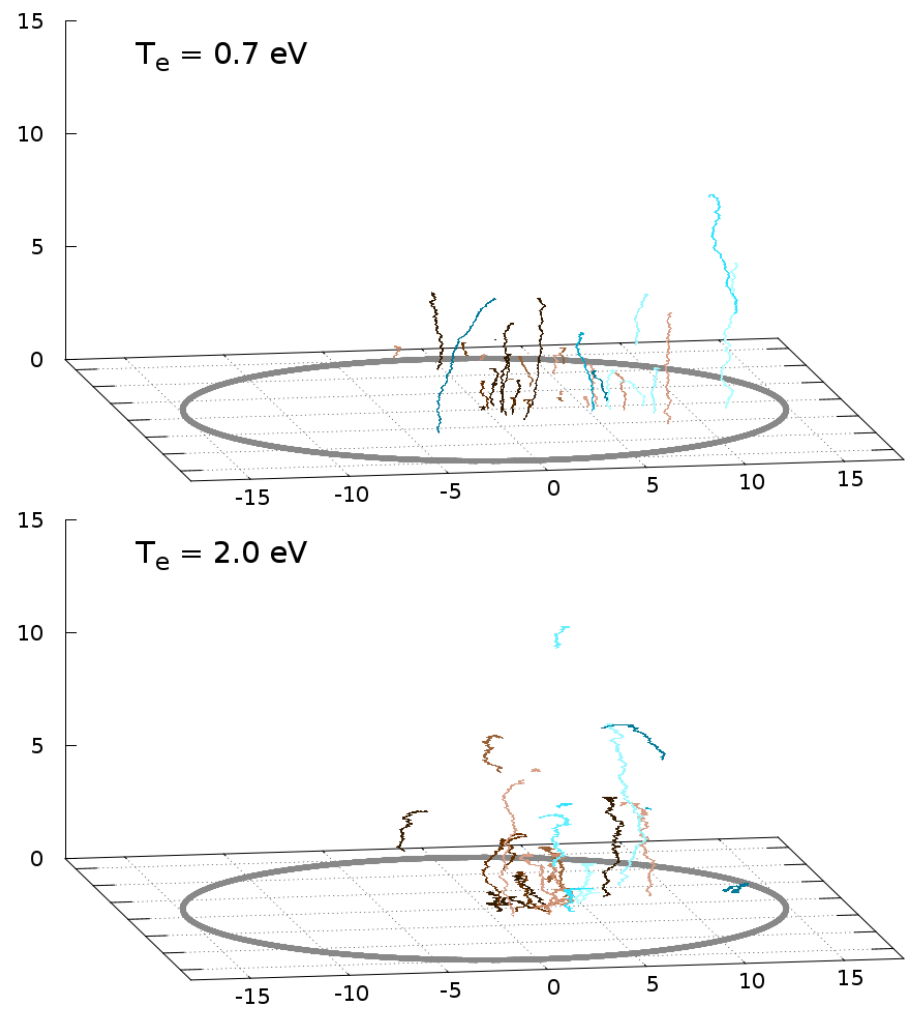

Figure 5. Simulated trajectories of particles eroded from a circular carbon target. The magnetic field points in the vertical direction, and the plasma axis is centered on the middle of the target. Test particles originating close to the center of the target are shown in browner colors, those originating closer to the edge are shown in bluer colors. The target is plotted as a grey circle. Results for two different electron temperatures are shown: $\mathrm{T}_{e}=0.7 \mathrm{eV}$ (top) and $\mathrm{T}_{e}=2.0 \mathrm{eV}$ (bottom). In both cases, $n_{e}=3.2 \cdot 10^{20} \mathrm{~m}^{-3}$.

Due to the high densities and low temperatures, the efficiency of Coulomb collisions between the ions and plasma ions is very high. This causes the impurity ions to follow a Brownian-like diffusive motion through the plasma, rather than a gyrating motion. The average impurity velocity approaches the plasma velocity very quickly.

At these plasma temperatures, the primary ionization process is charge exchange with the hydrogen plasma ions [14]. The primary recombination process of $\mathrm{CH}_{x}^{+}$is dissociative recombination. With $\mathrm{T}_{e}=0.7 \mathrm{eV}$, the rate of dissociative recombination is more than 2 times higher than for the $2 \mathrm{eV}$ case. Therefore, hydrocarbon molecules in the $0.7 \mathrm{eV}$ plasma spend less time as ions than hydrocarbons in the $2 \mathrm{eV}$ plasma. This is clearly visible in the plotted ion trajectories.

The radial electric field causes a rotation of the plasma that must be taken into account in the simulation: $\mathbf{v}_{\text {rot }}=\mathbf{E} \times \mathbf{B} / B^{2}$. If the plasma rotation would not be taken into account, then the applied electric field does not cause an $\mathbf{E} \times \mathbf{B}$ drift of the impurities, because the gyro-motion is continuously interrupted. Instead, impurity ions are then forced in the direction of the electric field. This causes a strong inwards transport; simulations without plasma rotation did indeed show a strong deposition peaking near the center of the target. However, once the plasma rotation is taken into 
account as well, the impurity ions are instead forced in the direction of the rotation; this is the $\mathbf{E} \times \mathbf{B}$-direction. This impurity rotation causes a Lorentz force in the $(\mathbf{E} \times \mathbf{B}) \times \mathbf{B}$ direction; this is the negative $\mathbf{E}$-direction. The resulting Lorentz force cancels out the inwards pointing electric force exactly. Thus, the radial E-field only causes impurity $\mathbf{E} \times \mathbf{B}$-drift, even in the high-collisionality regime.

To show the influence of the radial E-field, reference simulations were performed with and without any radial E-field or associated rotation. Figure 6 shows the resulting (simulated) redeposition distribution over the target for a set of carbon atoms that are each eroded at a single spot $10 \mathrm{~mm}$ away from the beam center, here for the $\mathrm{T}_{e}=2 \mathrm{eV}$ case. Both with and without the radial E-field, the deposition in the center of the target was larger than the deposition in the periphery. This is caused by the gradient in $n_{e}$. The higher density in the plasma center creates a higher ionization rate there, and thus the higher deposition rate at the target center. The radial electric field causes a clearly visible rotation of the impurity redeposition. In the $\mathrm{T}_{e}=0.7 \mathrm{eV}$ run, the relative influence of the radial E-field was less than in the plotted result because, due to the more efficient recombination, the electric force is acting on the hydrocarbons during a shorter time; the rotation in the deposition pattern was still present, but less pronounced than in the $\mathrm{T}_{e}=2 \mathrm{eV}$ simulation.
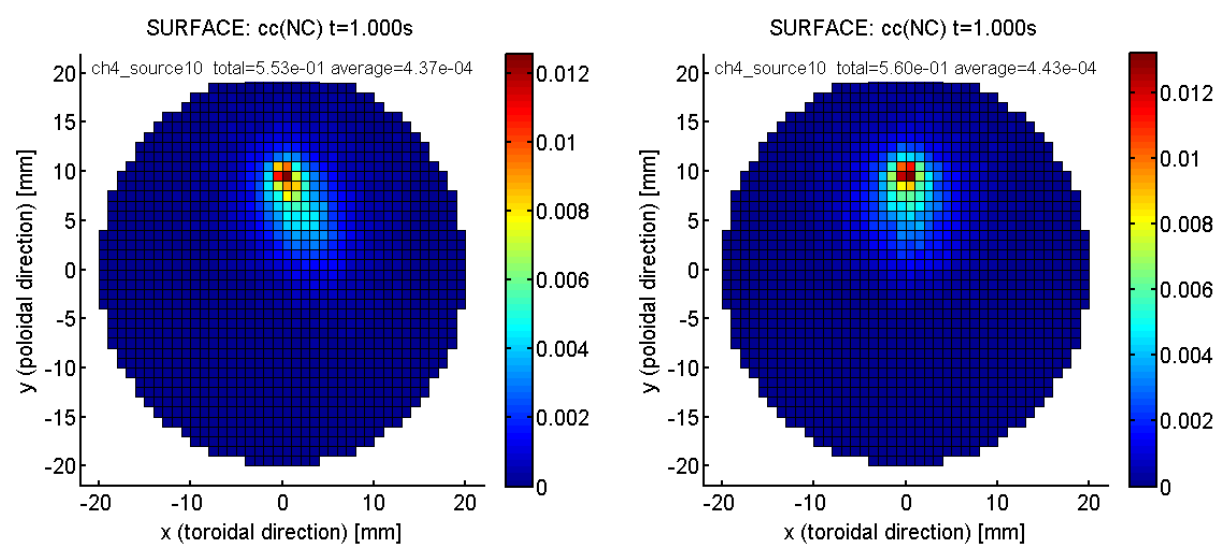

Figure 6. Distribution of returning carbon over the target, resulting from a demonstration run with molecules originating from a single spot $10 \mathrm{~mm}$ away from the beam center. In the left plot, the reference radial E-field peaking at $1.7 \mathrm{kV} / \mathrm{m}$ is assumed. In the right plot, no radial E-field is assumed. These simulations assumed $\mathrm{T}_{e}=2 \mathrm{eV}, \mathrm{n}_{e}=3.2 \cdot 10^{20} \mathrm{~m}^{-3}$.

An interesting observation is that the total return probability of eroded carbon is almost independent on the presence of a radial electric field. Only the location where the carbon returns is affected; both in the $\mathrm{T}_{e}=0.7 \mathrm{eV}$ runs and the $\mathrm{T}_{e}=2 \mathrm{eV}$ runs, the return probability was changed by less than $3 \%$ when the radial electric field was switched on. 


\section{Sticking probabilities}

In the previous section, the simulated locations of ion redeposition were presented. In order to infer the eroded layer thickness profile from this, it is necessary to know the probability that a hydrocarbon radical arriving at the surface is to be deposited there. This "sticking probability" is strongly dependent on the properties of the substrate. Important parameters affecting it include the impurity ion/radical energy, impurity species, surface composition and temperature, defect and dangling bond density, and surface roughness. Unfortunately, many of these are hard to determine accurately; in fact, they may even change during the experiment. Especially since we are operating in a new ion flux regime, this introduces uncertainty in the simulation result. Many different studies have been performed to obtain sticking probabilities (sometimes its inverse, the reflection probability, is given instead). A brief overview of results from literature for $\mathrm{CH}_{x}$ sticking on carbon surfaces will be given in this section.

First, however, it is necessary to know the kinetic energy of the impinging impurities in our experiment. In present experiments this is usually determined by the Debye sheath in front of the target, where a potential drop of approximately $3 k \mathrm{~T}_{e}$ accelerates the ions to the target. Following this potential drop, one might expect that in a typical Pilot-PSI plasma with $\mathrm{T}_{e}=1 \mathrm{eV}$, impurity ion energies at the surface are around $3 \mathrm{eV}$. However, typical impurity energies in Pilot-PSI are much higher, due to ion entrainment. Near the target, the plasma is accelerated to the sound speed, due to the Bohm sheath criterion. At the sheath edge, $v_{\text {plasma }}=\sqrt{k\left(T_{e}+3 T_{i}\right) / m_{H}} \approx 2 \sqrt{k T_{e} / m_{H}}$ (using the reasonable approximation that $T_{e} \approx T_{i}$ ). As the impurity ions approach the target, they are accelerated towards this velocity efficiently, due to the high collisionality. Crucially, however, a $\mathrm{CH}_{4}^{+}$ion is 16 times heavier than an $\mathrm{H}^{+}$ion. Therefore, if the $\mathrm{CH}_{4}$ ion fluid is fully thermalised with the hydrogen plasma, the impurity ion energy at the sheath edge follows:

$$
E_{C H 4}=\frac{1}{2} m v^{2}=2 \frac{m_{C H_{4}}}{m_{H}} k T_{e}=32 k T_{e}
$$

The energy spectrum of all neutral and ionic $\mathrm{CH}_{x}$ at the target was calculated by ERO for Pilot-PSI plasma with $\mathrm{T}_{e}=0.7 \mathrm{eV}$. It is shown in figure 7, with bin sizes of $1 \mathrm{eV}$. Not all impurities are completely thermalised with the plasma, resulting in a broad energy spectrum, roughly between 0 and $20 \mathrm{eV}$. The calculated average energy of all impurities was $8.1 \mathrm{eV}$. The low-energy peak at $E<1 \mathrm{eV}$ consists of the neutral $\mathrm{CH}_{4}$ that returns quickly, due to elastic collisions with plasma particles. Similar ion entrainment has been observed previously in the PISCES-A linear plasma generator, when running at relatively low $\mathrm{T}_{e}[9]$.

Knowing that the hydrocarbons have a broad energy spectrum between $0 \mathrm{eV}$ and $20 \mathrm{eV}$, we now evaluate the available data on sticking probabilities in this regime. The literature consists of experimental studies and molecular dynamics (MD) calculations.

Several experimental studies have been performed in the context of low-temperature plasma physics for deposition purposes. These studies focus on the $\mathrm{CH}_{3}$ radical (as well 


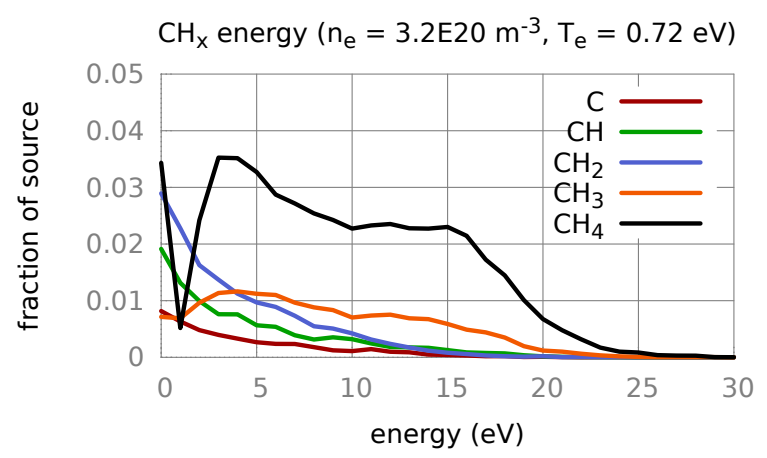

Figure 7. Energies of the returning hydrocarbon molecules, as calculated by ERO. This plot includes both ionic and neutral molecules. Plasma paramters: $n_{e}=$ $3.2 \cdot 10^{20} \mathrm{~m}^{-3}, \mathrm{~T}_{e}=0.72 \mathrm{eV}$.

as several $\mathrm{C}_{2} \mathrm{H}_{x}$ radicals), because low-temperature plasma-enhanced chemical vapour deposition (PECVD) experiments typically find this to be the most abundant radical emanating from methane plasmas [18]. However, these hydrocarbon radicals have nearthermal impact energies, so they lack bond-breaking energies. Furthermore, it was shown [19] that the sticking probability of hydrocarbon radicals depends strongly on the hybridization of the carbon atom carrying the dangling bond. This is in turn greatly influenced by the ion bombardment from the plasma: plasma surface effects, in particular ion bombardment and abstraction of surface-bonded $\mathrm{H}$ by incident $\mathrm{H}$-atoms determine the surface properties. Those processes will be different as the experimental conditions $\left(n_{e}, \mathrm{~T}_{e}\right.$, ion- and neutral fluxes) change. Therefore, experimental studies using low-temperature plasmas have only a very limited relevance to fusion-relevant surfaces. Jacob et al [19] have summarised the current best experimental knowledge; they are included in table 1.

The most relevant MD calculations found from the literature were:

- Tichmann et al have published energy-dependencies [20] and angular dependencies [21] of the sticking probabilities for $\mathrm{CH}_{x}(0 \leq x \leq 4)$ on $a-\mathrm{C}: \mathrm{H}$ films with properties identical to those experimentally obtained from real films. The temperature was held at $300 \mathrm{~K}$. These rates were used in previous ERO simulations [16] and will be used here as a reference.

- Sharma et al [22] simulated sticking on an $a$-C:H sample with an $\mathrm{H}$ :C ratio of 0.66 , at a temperature of $300 \mathrm{~K}$. They found the highest sticking rates of the studies cited here, approaching unity for all $\mathrm{CH}_{x}$ species for impact energies of $10 \mathrm{eV}$.

- Alman and Ruzic [23] used an amorphous graphite, focusing on sequential bombardement. Both a "hard" and a "soft" target were simulated; results from the "soft" layer are given here, due to the low density of the $a-\mathrm{C}: \mathrm{H}$ layer. They cite no surface temperature.

- De Rooij et al [24] calculated the reflection coefficient of $\mathrm{CH}_{x}$ on $a$-C:H samples; these samples were prepared by simulated exposure to various incident fluxes, and 
Erosion/re-deposition in an ITER-divertor-like plasma beam

Table 1. Sticking probabilities of different hydrocarbon molecules with $\mathrm{E}_{\text {impact }}=$ $10 \mathrm{eV}$, as published by different groups. See main text for descriptions of each of the studies.

Tichmann et al [20] (MD)

Sharma et al [22] (MD)

Alman and Ruzic [23] (MD)

De Rooij et al [24] (MD)

Ohya et al [25] (MD)

Jacob [19] (experiment, thermal energy)

$\begin{array}{lllll}\mathbf{C H}_{4} & \mathbf{C H}_{3} & \mathbf{C H}_{2} & \mathbf{C H} & \mathbf{C} \\ 0.03 & 0.62 & 0.86 & 0.92 & \text { n.a. } \\ >0.98 & >0.98 & >0.98 & >0.98 & >0.98 \\ 0.8 & 0.9 & 0.9 & >0.98 & 0.7 \\ \text { n.a. } & 0.3-0.5 & 0.5-0.8 & 0.6-0.8 & 0.6-0.8 \\ <0.02 & 0.07 & 0.26 & 0.79 & 0.90 \\ \text { n.a. } & 0.01 & 0.025 & 1 & 1\end{array}$

had two different surface temperatures (700 K and $1000 \mathrm{~K}$ ).

- Ohya et al [25] have calculated the reflection coefficient of $\mathrm{CH}_{x}$ on hydrogenated and amorphized graphite, and performed ERO simulations with the resulting rates, citing no surface temperature. They used the ternary W-C-H potential from Juslin et al [26]; this is in contrast to all the other studies cited here, which use the empirical potential from Brenner [27].

The results at $\mathrm{E}_{\text {impact }}=10 \mathrm{eV}$ are summarised in table 1 .

It should be noted that a strong impact energy dependence is found in all MD simulations: the lower-energy ions have a much lower sticking probability. Yet, the results clearly do vary quite strongly, dependent on the simulation technique employed. In the next section, several sets of sticking rates will be compared to get an indication of the error introduced by this uncertainty.

\section{Integrated erosion/redeposition simulations}

Using our ERO simulation, we calculated the expected erosion profile of the $a$-C:H layer after 10 seconds of exposure to the Pilot-PSI plasma. Making use of the cylindrical symmetry, the target surface was divided in 20 annular bins with radii $\mathrm{d} r=1 \mathrm{~mm}$, centered around the center of the plasma column. The erosion flux $\Gamma_{E R}$ is calculated at every bin, using the incident ion flux $\Gamma_{H}$ and the erosion yield $Y$ :

$$
\Gamma_{E R}(r)=\Gamma_{H}(r) \cdot Y
$$

Erosion from a bin continues until the layer is depleted; the initial layer thickness is equal to the experimentally measured layer thickness outside the beam center. The redeposition is calculated using ERO simulation; this process is described in [16]. After iteration over many (small) time steps, the net erosion profile is obtained.

Given the magnitude of the differences between sticking rates found by different authors (described in section 4), several sets of sticking rates were compared. The rates from Tichmann et al [20] are used as a reference; the sticking rates found by Sharma et al [22] were the highest and are used as "upper bound" values; similarly, the rates from Ohya et al were used as "lower bound" values. The chemical erosion yield $Y$ depends on 

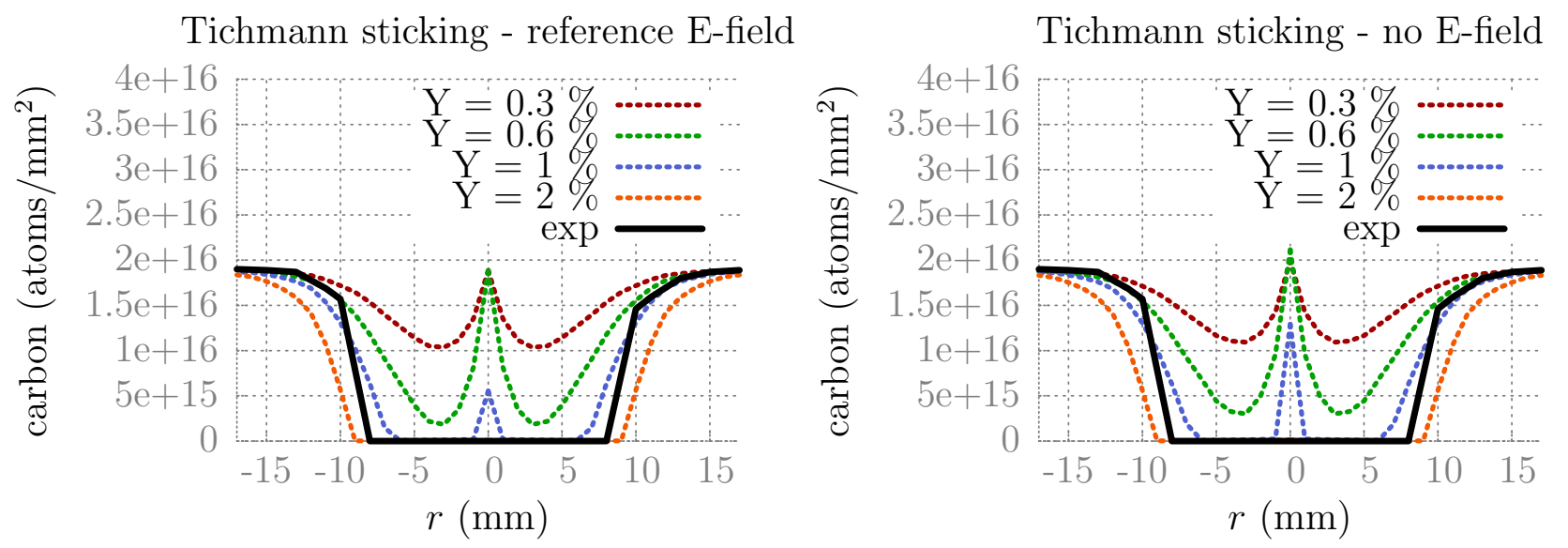

Figure 8. Experimental erosion profiles (black line), and simulation results with various erosion yields (dashed lines). In the upper plot, the reference radial E-field was taken into account in the calculations; in the lower plot it was disabled. The sticking probabilities are taken from Tichmann et al [20].

many factors, for example the surface temperature, H:C ratio, dangling bond density, and the hydrogen ion flux and energy. The carbon erosion yield in the present regime has been studied previously [28, 29], but very large uncertainties remain. Therefore it will be treated here as one of the unknowns in the simulation. For simplicity, it is assumed to be constant along the surface. Due to gradients in incoming plasma flux, this assumption is not guaranteed to hold. However, this does allow us to make a rough comparative analysis of the influence of various parameter variations.

The resulting layer thicknesses calculated using the sticking coefficients from Tichmann et al [20] (see section 4) are shown in figure 8. The different dashed lines are simulations with different erosion yields, as indicated in the legend. For comparison, the experimental result is shown by the black line. At $Y<2 \%$, there is a clear net deposition peak at the center of the target. This is because of the density and temperature gradient: the ionization rate is higher in the center of the plasma column, therefore redeposition is strongest there. Experimentally, this peak is not observed, as the entire layer was eroded away in the target center. The same occured in the simulations with $Y \geq 2 \%$.

Although the radial electric field caused a significant rotation of the re-deposited impurities, it did not have a strong influence on the redeposition pattern. The only effect was a slight reduction of the deposition peak in the center of the target. This slight reduction seems to be caused by the rotation, which hampers transport of material from the target periphery to its center. It is important to realize that, when doing any impurity transport simulations, an E-field perpendicular to the magnetic field must always be accompanied by the corresponding plasma drift velocity in the $\mathbf{E} \times \mathbf{B}$-direction, and vice versa. Omitting this plasma drift resulted in an unphysical impurity drift in the direction of $E_{\perp}$. The cause for this drift was the high collisionality, which prevents gyromotion in the $\mathbf{E} \times \mathbf{B}$-direction. The magnitude of the perpendicular electric field in ITER is, as yet, quite uncertain [30]; however, the results here suggest that this uncertainty 

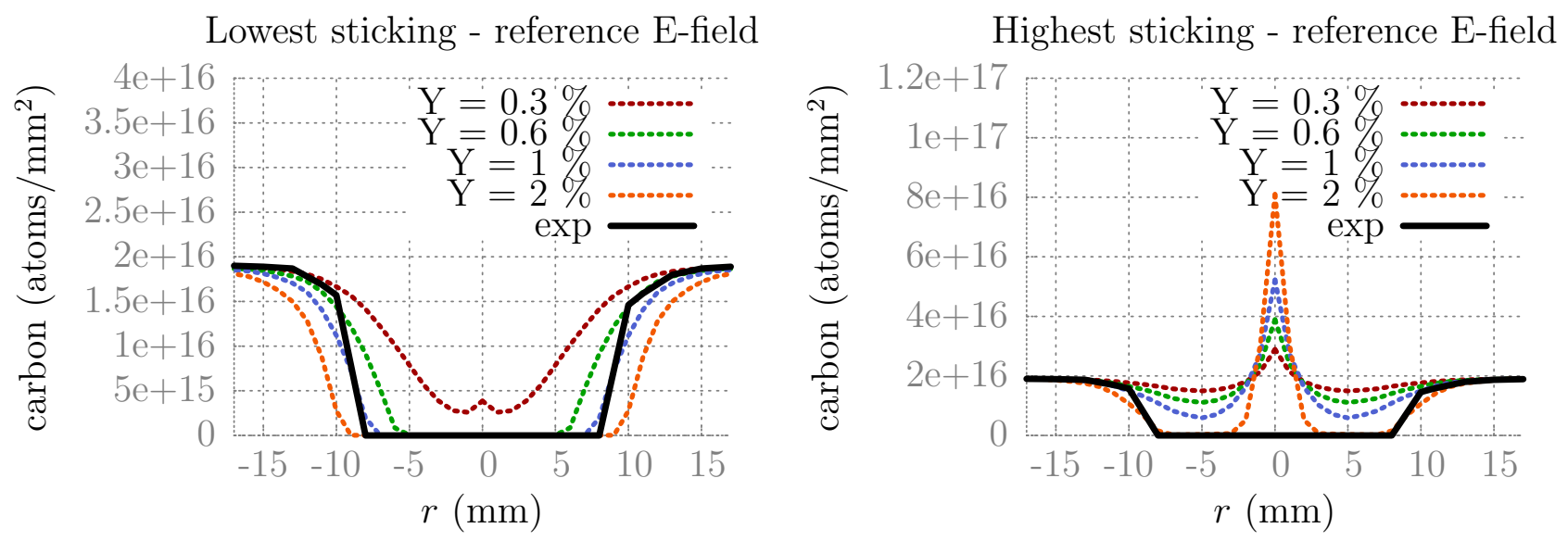

Figure 9. Erosion profiles, assuming sticking coefficients from Ohya et al [25] (above) and from Sharma et al [22] (below). The reference radial E-field was used here.

does not have an enormous impact on the impurity transport in the $E_{\perp}$-direction.

To study the influence of the sticking factor uncertainty, the calculations were repeated using first the lowest and then the highest sticking coefficients found in the literature. Results are shown in figure 9. The higher sticking rate from Sharma et al leads to a very strong deposition peaking that does not match the experiment at all. With the lower sticking rate, the deposition peaking in the center of the target is strongly reduced; deposition profiles acquired in this way actually match the experiment fairly well. Interestingly, it was found in TEXTOR that re-deposited layers can be re-eroded again very easily due to the impinging hydrogen flux [31]. There, this process reduced the effective hydrocarbon sticking rate quite severely. It is very well possible that such a process might be at play under these plasma conditions, too. However, conclusive evidence for that hypothesis is lacking.

With both the rates from Ohya et al and the rate from Tichmann et al, the experimental result curve lies in between the simulations with $Y=0.6 \%$ and $Y=2 \%$. This suggests that the actual gross erosion yield was in between those two values. Those yields fall well inside the range of erosion yields for graphite that have been observed under similar conditions in Pilot-PSI [29].

\section{Conclusion}

Hydrocarbon impurity erosion-transport-redeposition simulations with the 3D Monte Carlo code ERO were benchmarked against experiments in a high- $n_{e}$, low- $T_{e}$ plasma produced at the Pilot-PSI linear plasma generator. The ion-ion collisionality is proportional to $n_{i} \cdot T_{i}^{-3 / 2}$; it is very high at the densities that are achieved in PilotPSI and expected in ITER. Ions therefore do not complete Larmor orbits, but rather follow diffusive trajectories through the plasma. The impact energy spectrum of the $\mathrm{CH}_{x}$ molecules impinging on the surface was broadly distributed between 0 and $20 \mathrm{eV}$, 
averaging $8.1 \mathrm{eV}$. This was much higher than the electron temperature of $0.7 \mathrm{eV}$, because of entrainment of the heavier hydrocarbons with the light $\mathrm{H}^{+}$ions.

Due to the high ion-ion collisionality, one might expect that impurity ions would be pulled in the direction of an $\mathbf{E}$-field component perpendicular to $\mathbf{B}$, rather than in the $\mathbf{E} \times \mathbf{B}$-direction. However, it was shown that the radial electric field in Pilot-PSI does not affect the calculated re-deposition significantly. Even in high-collisionality plasmas, a perpendicular electric field causes only an $\mathbf{E} \times \mathbf{B}$ drift of the impurities, if the plasma drift velocity is correctly taken into account. The impurity transport simulations did show transport of eroded hydrocarbon ions toward the center of the Pilot-PSI beam. However, this was due to the density and temperature gradients of the plasma, and not due to the inwards pointing electric field. This conclusion is similar to the one drawn from analysis of tungsten migration in the C-MOD divertor [7] at a lower collisionality $\left(\mathrm{T}_{e}>5 \mathrm{eV}\right.$ and $n_{e} \leq 10^{20} \mathrm{~m}^{-3}$ ): there, too, the difference between runs with and without a radial electric field was small.

An overview has been made of various published results for the sticking of

hydrocarbon molecules with $\mathrm{E}_{\text {impact }}=10 \mathrm{eV}$ on an $a$-C:H surface. In combination with these sticking probabilities, the calculated inwards impurity transport did lead to a prediction of net deposition in the center of the plasma, for erosion yields below $2 \%$. This was not observed experimentally. Comparison of simulations with different sticking probabilities shows that the uncertainty in this probability may easily explain this discrepancy. It is possible that if the erosion period would have been shorter, a deposition area may have remained in the center of the target, but experimentally, the ramp-up and ramp-down time of Pilot-PSI made testing this difficult.

\section{Acknowledgments}

This work was supported by the European Communities under the contract of Association between EURATOM/FOM and carried out within the framework of the European Fusion Programme with financial support from NWO and the NWO Grant No. RFBR 047.018.002. The views and opinions expressed herein do not necessarily reflect those of the European Commission.

\section{References}

[1] M. Mayer et al. J. Nucl. Mat., 337-339:119-123, 2005.

[2] G. Federici et al. J. Nucl. Mater., 313-316(0):11-22, 2003.

[3] A. Loarte et al. Iter physics basis, chapter 4. Nucl. Fusion, 47:S203-S263, 2007.

[4] J D Huba. NRL plasma formulary, page 33. Naval Research Laboratory, 2009.

[5] H. J. N. van Eck, A. W. Kleyn, H. J. van der Meijden, G. J. van Rooij, J. Scholten, and P. A. Zeijlmans van Emmichoven. Appl. Phys. Lett., 101(22):224107.

[6] G. J. Van Rooij et al. Appl. Phys. Lett., 90:121501, 2007. 
[7] J. N. Brooks. Nucl. Fusion, 53:042001, 2013.

[8] P. L. Stangeby. J. Phys. D, 20:1472, 1987.

[9] E. M. Hollmann, K. R. Umstadter, R. P. Doerner, J. Munoz, D. Nishijima, and A. Yu. Pigarov. J. Nucl. Mater., 415:S425-S429, 2011.

[10] A. Kirschner, D. Borodin, S. Droste, V. Philipps, U. Samm, G. Federici, A. Kukushkin, and A. Loarte. J. Nucl. Mater., 363-365:91-95, 2007.

[11] D. Borodin et al. Contrib. Plasma Phys., 50(3-5):432-438, 2010.

[12] J. W. A. M. Gielen, P. R. M. Kleuskens, M. C. M. van de Sanden, L. J. van IJzendoorn, D. C. Schram, E. H. A. Dekempeneer, and J. Meneve. J. Appl. Phys., 90(10):5986.

[13] W. Jacob. Thin Solid Films, 326(1-2):1-42, 1998.

[14] R.K. Janev and D. Reiter. Phys. Plasmas, 9, 2002.

[15] G.A. van Swaaij et al. Plasma Phys. and Control. Fusion, 54(9), 2012.

[16] G.A. Van Swaaij, K. Bystrov, D. Borodin, A. Kirschner, T. Zaharia, and L.B Van der Vegt. http://dx.doi.org/10.1016/j.jnucmat.2013.01.132, 2013.

[17] A. E. Shumack, H. J. De Blank, J. Westerhout, and G. J. Van Rooij. Plasma Phys. Control. Fusion, 54(12):125006, 2012.

[18] M. Shiratani, J. Jolly, H. Videlot, and J. Perrin. Jpn. J. Appl. Phys., 36(7 SUPPL B):4752-4755, 1997.

[19] W. Jacob. J. Nucl. Mater., 337-339:839-846, 2005.

[20] K. Tichmann, U. von Toussaint, and W. Jacob. J. Nucl. Mater., 420:291-296, 2011.

[21] K. Tichmann, U. von Toussaint, and W. Jacob. J. Nucl. Mat., 514:S196-S199, 2011.

[22] A. R. Sharma et al. J. Nucl. Mater., 363-365:1283-1288, 2007.

[23] D. A. Alman and D. N. Ruzic. Phys. Scr., T111:145-151, 2004.

[24] E. D. De Rooij, A. W. Kleyn, and W. J. Goedheer. Phys. Chem. Chem. Phys., 12:14067-14075, 2010.

[25] L. Ohya, Y. Kikuhara, K. Inai, A. Kirschner, D. Borodin, A. Ito, H. Nakamura, and T. Tanabe. J. Nucl. Mater., 390-391:72-75, 2009.

[26] N. Juslin et al. J. Appl. Phys., 98(12):123520, 2005.

[27] D. W. Brenner. Phys. Rev. B, 42(15):9458, 1990.

[28] J. Roth et al. J. Nucl. Mater, 337-339:970, 2005.

[29] J. Westerhout. Carbon Chemical Erosion in High Flux and Low Temperature Hydrogen Plasma. PhD thesis, Technical University Eindhoven, 2010.

[30] A. Loarte et al. Nucl. Fusion, 47:S203-S263, 2007.

[31] A. Kirschner et al. J. Nucl. Mater., 415(1 SUPPL):S239 - S245, 2011. 